SHORT REPORT

\title{
Whole body muscle hypertrophy from resistance training: distribution and total mass
}

\author{
T Abe, K Kojima, C F Kearns, H Yohena, J Fukuda
}

Br J Sports Med 2003;37:543-545

Objective: To examine the absolute and relative changes in skeletal muscle (SM) size using whole body magnetic resonance imaging (MRI) in response to heavy resistance training (RT).

Method: Three young men trained three days a week for 16 weeks.

Results: MRI measured total SM mass and fat free mass (FFM) had increased by $4.2 \mathrm{~kg}$ and $2.6 \mathrm{~kg}$ respectively after resistance training.

Conclusions: RT induces larger increases in SM mass than in FFM. RT induced muscle hypertrophy does not occur uniformly throughout each individual muscle or region of the body. Therefore the distribution of muscle hypertrophy and total SM mass are important for evaluating the effects of total body RT on muscle size.

A ccurate measurements of skeletal muscle (SM) mass and distribution in humans are important for studies of SM hypertrophy response to heavy resistance training (RT). Currently, the most accurate in vivo methods of measuring SM mass are multiscan magnetic resonance imaging (MRI) and computed tomography. ${ }^{1}$ Despite its safety, most MRI studies have only evaluated regional-for example, arms, trunk, and legs-SM mass. ${ }^{1}$ We recently reported whole body MRI using a contiguous slice by slice (no interslice gap) method to evaluate total SM mass and its distribution. ${ }^{2}$ Using this approach, the distribution of RT induced whole body SM hypertrophy can be investigated.

To date, most studies ${ }^{3}{ }^{4}$ have only evaluated limb muscle hypertrophy, and very few have reported RT induced muscle hypertrophy in the trunk region. ${ }^{5}$ More importantly, the distribution of the relative increases in RT induced muscle hypertrophy has not been reported. Thus the purpose of this pilot study was to examine the absolute and relative changes in SM size using contiguous whole body MRI scans in response to RT.

\section{METHODS}

Three healthy young men (age 20-21 years) volunteered for the study. All were physically active, but none had participated in RT before the start of the programme. All subjects signed informed consent documents. The department's ethical commission approved the study.

RT was carried out three days a week for 16 weeks. Three lower body (squat, knee extension, and knee flexion) and two upper body (bench press and latissimus dorsi pull down) exercises were performed. Workouts consisted of a warm up set followed by three sets to failure of 8-12 repetitions for each of the five exercises. The loads were progressively increased to maintain this range of repetitions per set. One repetition maximum (IRM) strength was determined by progressively increasing the weight lifted until the subject
Table 1 Body composition and strength before and after training

\begin{tabular}{llllll}
\hline & Subject & Before & After & Difference & Change (\%) \\
\hline Body mass (kg) & A & 62.9 & 65.0 & 2.1 & 3.3 \\
& B & 59.1 & 61.3 & 2.2 & 3.7 \\
& C & 65.2 & 67.9 & 2.7 & 4.1 \\
FFM $(\mathrm{kg})$ & Mean & 62.4 & 64.7 & 2.3 & 3.7 \\
& A & 55.1 & 57.2 & 2.1 & 3.8 \\
& B & 53.5 & 55.6 & 2.1 & 3.9 \\
Total SM mass & M & 59.9 & 63.4 & 3.5 & 5.8 \\
(kg) & Aean & 56.1 & 58.7 & 2.6 & 4.5 \\
& B & 20.5 & 24.6 & 4.1 & 20.0 \\
BP strength (kg) & Mean & A.6 & 24.7 & 5.1 & 26.0 \\
& C & 25.7 & 28.6 & 3.5 & 13.9 \\
& B & 40.5 & 57.0 & 4.2 & 19.4 \\
& C & 60.0 & 55.0 & 15.0 & 35.3 \\
& Mean & 47.5 & 60.8 & 10.0 & 37.5 \\
& & & 13.3 & 29.7 \\
\hline
\end{tabular}

BP, Bench press; FFM, fat free mass; SM, skeletal muscle.

failed to lift the weight through a full rage of motion. Strength of the squat was assessed using the 3RM test.

Total body SM distribution and mass were measured using an MRI 1.5-T scanner (GE Signa, Milwaukee, Wisconsin, USA) with spin echo sequence (TR, 1500 milliseconds; TE, 17 milliseconds). ${ }^{2}$ Contiguous transverse images with $1.0 \mathrm{~cm}$ slice thickness (no interslice gap) were obtained from the first cervical vertebra to the ankle joints for each subject. Four sets extended from the first cervical vertebra to the femoral head during breath holding (about 20 seconds). The other three sets of acquisitions were obtained from the femoral head to the ankle joints during normal breathing. In each slice, the cross sectional area (CSA) was digitised, and the muscle tissue volume $\left(\mathrm{cm}^{3}\right)$ per slice was calculated by multiplying the CSA $\left(\mathrm{cm}^{2}\right)$ by slice thickness $(\mathrm{cm})$. SM volume units (litres) were converted into mass units $(\mathrm{kg})$ by multiplying the volumes by the assumed constant density for SM $(1.041 \mathrm{~kg} / \mathrm{l}){ }^{6}$

Body density was measured by hydrostatic weighing with simultaneous measurement of residual lung volume by oxygen dilution. Body fat percentage was calculated from body density using the equation of Brozek et al. ${ }^{7}$ Fat free mass (FFM) was estimated as body mass minus fat mass.

\section{RESULTS}

Mean relative increases in upper body and lower body strength (IRM or 3RM) after RT were $30 \%$ and $16 \%$ respectively. Body fat decreased by $0.6 \%$ on average, and

Abbreviations: CSA, cross sectional area; MRI, magnetic resonance imaging; RT, resistance training; SM, skeletal muscle; FFM, fat free mass 


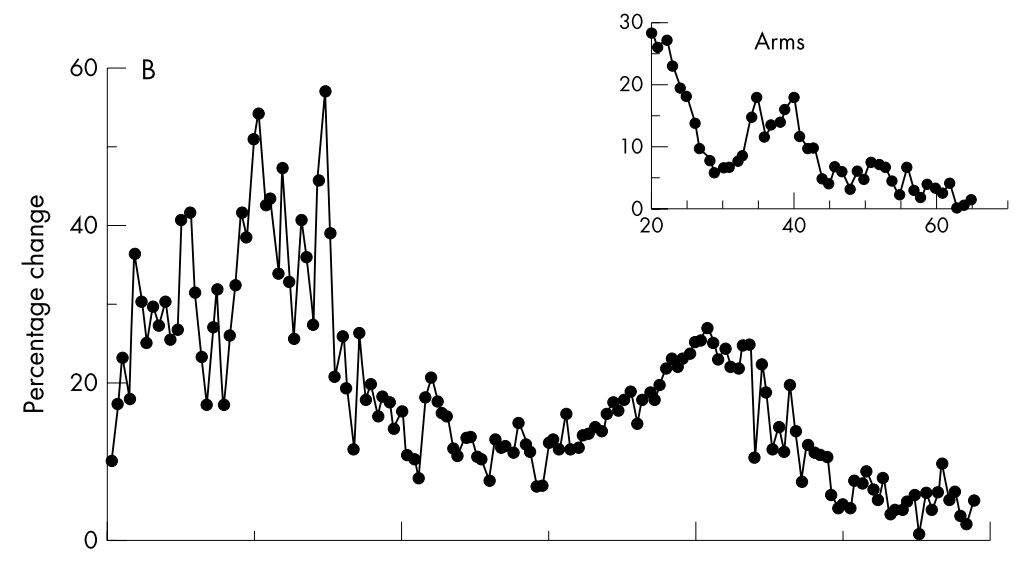

Figure 1 Absolute and relative changes in skeletal muscle size distribution in response to total body resistance training. CSA, Cross sectional area.
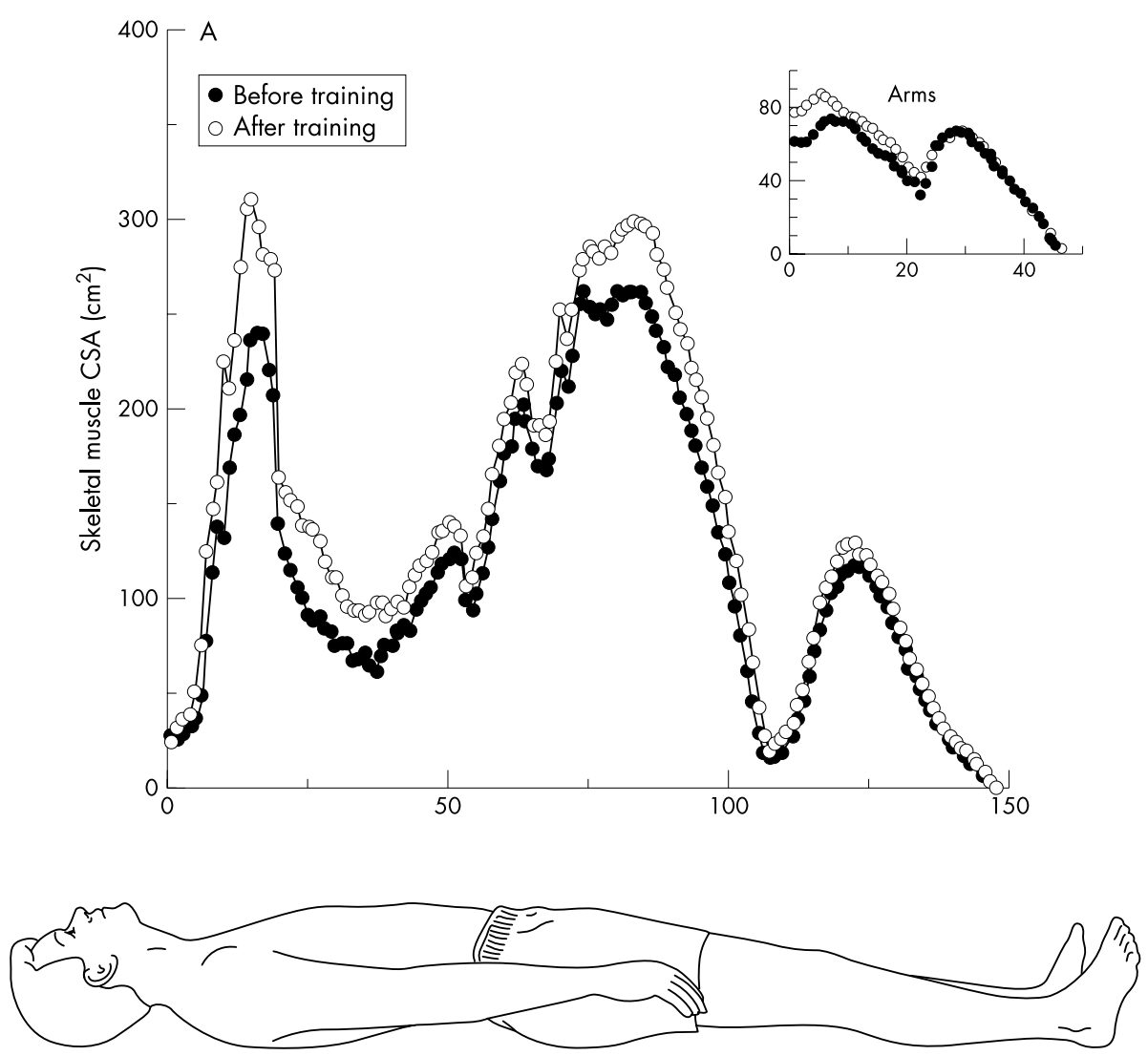

FFM increased by $2.6 \mathrm{~kg}$ after RT. The mean increase in total SM mass after RT was $4.2 \mathrm{~kg}$ (table 1 ).

The greatest absolute increases in muscle CSA were seen at the level of the shoulder, chest, upper thigh, and upper portion of the upper arm (fig 1A; subject A). Relative changes in muscle hypertrophy were greater at the level of the shoulder, chest, and upper portion of the upper arm $(+25-$ $40 \%)$ compared with the waist, hip, forearm, thigh, and lower leg (+10-20\%) (fig 1B). The relative increase in muscle CSA of all three subjects was $26 \%$ at the shoulder (peak CSA level) and $18 \%$ and $9 \%$ at the mid-thigh and lower leg respectively.

\section{DISCUSSION}

It has been reported that FFM increases by about $2.0 \mathrm{~kg}$ after 10-16 weeks of total body RT. ${ }^{3}$ However, very little is known about the degree of SM increase after RT. The mean increase in SM in this study was $4.2 \mathrm{~kg}$. Nelson and coworkers ${ }^{8}$ reported a $1.4 \mathrm{~kg}$ (24 hour urinary creatinine) and $1.6 \mathrm{~kg}$ (in vivo neutron activation) increase in total SM in postmenopausal women after 52 weeks of randomised controlled RT. Although the SM gain in our study was threefold higher than in other reports, ${ }^{8}$ the relative increases in limb muscle CSA were consistent with the literature (5$10 \%$ increase in lower body and $15-30 \%$ increase in upper body muscle CSA after 12-16 weeks of RT). ${ }^{34}$ Nelson et al, ${ }^{8}$ on the other hand, only reported a $6-8 \%$ increase in arm and thigh muscle CSA. The differences in RT induced SM gain between our data and other reports are probably due to differences in the training programmes-for example, training frequency.

The novel finding of this study was that the RT induced increase in total SM mass measured by MRI was larger than the increase in FFM. Another study ${ }^{8}$ showed decreases in non-SM lean tissue (as measured by in vivo neutron activation) and increases ${ }^{8}$ or decreases ${ }^{9}$ in total body water 


\section{Take home message}

Resistance training induces larger increases in skeletal muscle mass than in fat free mass. Muscle hypertrophy does not occur uniformly throughout each individual muscle or region of the body.

after RT. In our study, there were no differences in total body water (bioelectrical impedance analysis method) after training (63.6-69.3\% before $v$ 63.4-67.5\% after). One possible explanation is that non-SM lean tissue may decrease after RT. Clearly, more work is needed to determine if there are changes in organ or non-SM lean tissue after RT.

If changes in muscle hypertrophy were constant across every muscle, then a single anatomical CSA would reflect changes in SM mass. However, our data show that muscle hypertrophy did not occur uniformly throughout each individual muscle or region-for example, trunk, arm, and leg-of the body. Therefore the distribution of muscle hypertrophy and SM mass are important for evaluating the effects of total body RT because there are differences between relative changes in individual muscle CSA and SM mass.

\section{ACKNOWLEDGEMENTS}

We extend our gratitude to the subjects who participated in this study and to Ms Sumie Komuro for her assistance. This study was supported in part by The Ministry of Education, Science, Sports and Culture of Japan (grant No 15300221).

\section{Authors' affiliations}

T Abe, K Kojima, Tokyo Metropolitan University, Exercise and Sport

Science, Minami-Ohsawa, Hachioji, Tokyo 192-0397, Japan

C F Kearns, Schering-Plough Research Institute, Kenilworth, NJ 07033-

1300, USA

H Yohena, J Fukuda, Fujisawa-Shonandai Hospital, Fujisawa,

Kanagawa, Japan

Correspondence to: Dr Abe, Tokyo Metropolitan University, Exercise and Sport Science, Minami-Ohsawa, Hachioji, Tokyo 192-0397, Japan; abebe@comp.metro-u.ac.jp

Accepted 11 February 2003

\section{REFERENCES}

1 Janssen I, Heymsfield SB, Wang Z, et al. Skeletal muscle mass and distribution in 468 men and women aged 18-88 yr. J Appl Physiol 2000:89:81-8.

2 Abe T, Kearns CF, Fukunaga T. Sex differences in magnetic resonance imaging measured whole body skeletal muscle mass and distribution in young Japanese adults. Br J Sports Med 2003;37:436-40.

3 Cureton KJ, Collins MA, Hill DW, et al. Muscle hypertrophy in men and women. Med Sci Sports Exerc 1988;20:338-44.

4 Abe T, DeHoyos DV, Pollock ML, et al. Time course for strength and muscle thickness changes following upper and lower body resistance training in men and women. Eur J Appl Physiol 2000;81:174-80.

5 Danneels LA, Vanderstraeten GG, Cambier DC, et al. Effects of three different training modalities on the cross sectional area of the lumber multifidus muscle in patients with chronic low back pain. Br J Sports Med 2001;35:186-91.

6 Snyder WS, Cooke MJ, Manssett ES, et al. Report of the Task Group on Reference Man. Oxford: Pergamon, 1975:112.

7 Brozek J, Grande F, Anderson JT, et al. Densitometric analysis of body composition: revision of some quantitative assumption. Ann NY Acad Sci 1963; 110:113-40.

8 Nelson ME, Fiatarone MA, Layne JE, et al. Analysis of body-composition techniques and models for detecting change in soft tissue with strength training. Am J Clin Nutr 1996;63:678-86.

9 Campbell WW, Joseph UO, Davey SL, et al. Effects of resistance training and chromium picolinate on body composition and skeletal muscle in older men. J Appl Physiol 1999;86:29-39.

\section{How valid is a self reported 12 month sports injury history?}

\section{B J Gabbe, C F Finch, K L Bennell, H Wajswelner}

Background: A past injury history is one of the most commonly cited risk factors for sports injury. Often, injury history data are collected by self report surveys, with the potential for recall bias.

Objective: To assess the accuracy of a 12 month injury history recall in a population of 70 community level Australian football players.

Methods: The retrospective, self reported injury histories of 70 community level Australian football players were compared with prospective injury surveillance records for the same 12 month period. The accuracy of the players' recall of the number of injuries, injured body regions, and injury diagnosis was assessed.

Results: Recall accuracy declined as the level of detail requested increased. All players could recall whether or not they were injured during the previous year. Almost $80 \%$ were able to accurately recall the number of injuries and body regions injured, but not the diagnoses, whereas only $61 \%$ were able to record the exact number, body region, and diagnosis of each injury sustained.

Discussion: The findings of this study highlight the difficulty of using retrospectively collected injury data for research purposes. Any injury research relying on self reported injury history data to establish the relation between injury history and injury risk should consider the validity of the self report injury histories.
$\mathrm{C}_{\text {hist }}$ ne of the most commonly reported risk factors for sports injury is the presence of a positive past injury history. ${ }^{1-6}$ However, often this is based on self reported data, relying on the participants' correct memory of events. This reliance on memory can introduce recall bias, ${ }^{78}$ potentially leading to incorrect conclusions about the epidemiology of sports injuries sustained and the relation between past and future injury.

The potential for recall bias can be avoided altogether if self reported injury data are avoided. For example, information could be extracted from a participant's medical record or from prior injury surveillance records. ${ }^{7}$ However, difficulties arise with respect to accessing medical record data for establishing an injury history. Sports participants can seek treatment from more than one type of health professional and in more than one location, increasing the difficulty of collecting the relevant information. In addition, continuing sports injury surveillance systems using prospective methods are relatively uncommon, particularly in Australia. Prospective studies are often time consuming and can be expensive to undertake because of the length of data collection and the degree of monitoring involved. ${ }^{10}$ Therefore, studies designed to evaluate the relation between an injury history and a subsequent injury must often rely on self reported data.

Minimisation of recall bias is a prerequisite when the collection of self reported data cannot be avoided. ${ }^{7}$ Providing 


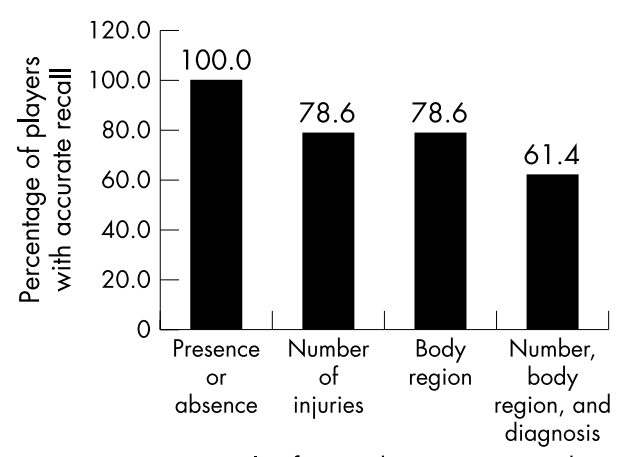

Details of injury history requested

Figure 1 Accuracy of player recall of aspects of a 12 month injury history.

a clear definition of injury can help to improve the memory of participants through the provision of specific prompts. For example, requesting information about injuries resulting in a designated number of missed training sessions or games is likely to result in better recall than simply asking about any pain experienced. ${ }^{11}$

Limiting the length of time over which participants are asked to recall injuries can also help to reduce the impact of recall bias. ${ }^{12}$ The longer the time frame, the less likely the injury history is to be accurate. Previous sports injury studies have tended to focus on a 12 month injury history, ${ }^{23613}$ potentially to ensure that all injuries sustained during the previous season are included. However, the validity of a sports participant's recall of injuries sustained over this time frame has not been well established.

Twellaar $e t a l^{14}$ found that sports participants recalled $61 \%$ of their injuries accurately one year later, but the injury definition used was very broad and included "any episode the athlete reported". Whether this injury definition was sufficient to stimulate accurate recall in athletes 12 months after injury is questionable. However, a 39\% drop off in injury recall accuracy from two to 12 months has also been shown in a study of work related farming injuries requiring medical treatment. $^{12}$

A study comparing retrospective and prospective injury data from a population of gymnasts found a higher incidence of injuries when data were collected prospectively. ${ }^{15}$ However, the comparison data were from two different time periods and therefore the accuracy of the retrospective data could not be established.

This study describes the accuracy of a 12 month self reported injury history in a group of community level Australian football participants.

\section{METHODS}

During the 1999 Victorian Amateur Football Association season, 350 football players participated in a prospective injury surveillance study. This study collected information about all football related injuries resulting in missed participation time and/or treatment from a health professional on a standardised data collection form. The club physiotherapist was responsible for injury data collection, and training and match exposure were collected by the coaching staff. The results of this study and the methodology used are published elsewhere. ${ }^{16}$ All injuries resulting in missed participation time and/or treatment from a health professional were recorded by the surveillance system prospectively.

In 2000, a prospective cohort study of risk factors for lower limb injuries was undertaken. Eighty five players in this risk factor study were also participants of the 1999 injury surveillance study.

Players participating in the 2000 study were required to recall any injuries they sustained during the previous football season-that is, during 1999-that resulted in them missing two or more training sessions and/or one or more games, and/ or requiring treatment from a health professional. The number of injuries sustained, body region injured, and the diagnosis of the injuries were requested. The questionnaire was completed just before the start of the competitive season during a club training session in the presence of a data collector. The questionnaire was based on one used previously in a case-control study of risk factors for injury in Australian football ${ }^{17}$ and was pilot tested before implementation.

To assess the accuracy of the 12 month self reported injury history, these recall data were compared with the prospectively collected injury surveillance data from the previous season in players that participated in both studies. The comparison was made on only those injuries that met the criterion of the 2000 survey, as the injury surveillance definition was slightly broader during the 1999 surveillance because of the inclusion of all injuries resulting in missed playing time rather than those resulting in two or more missed training sessions and/or one or more missed games. The accuracy of the players' recall of the number of injuries, injured body regions, and injury diagnosis was determined by comparing the self reported injury history with the injury surveillance records from the previous season. The level of agreement between the two was calculated as the proportion of instances in which the two records agreed. Where possible, $\kappa$ tests were performed to establish the degree of agreement between retrospective and prospective data. ${ }^{18}$ The $95 \%$ confidence interval (CI) was calculated for $\kappa \cdot \chi^{2}$ analysis was used to test for an association between the retrospective recall accuracy of players and the number of injuries recorded prospectively.

\section{RESULTS}

Of the 85 players eligible for the comparison, only $70(82 \%)$ completed a baseline risk factor questionnaire and are therefore included in these analyses.

Perfect agreement ( $\kappa=1.00,95 \%$ CI 1.00 to 1.00 ) was shown between retrospective and prospective records when players were asked whether or not an injury had been sustained during the previous season-that is, yes or no. Fifteen players were not injured in the previous season according to prospective records, and all confirmed this in the retrospective survey.

Figure 1 illustrates the proportion of players who were able to accurately recall the various aspects of their injury history.

Fifty five players (79\%) accurately recalled the number of injuries sustained during the previous football season, showing good agreement between retrospective and prospective records $(\kappa=0.71,95 \%$ CI 0.57 to 0.84$)$. The same number accurately reported the injured body regions. Only $61 \%$ of players could accurately recall the injury diagnosis, the number of injuries sustained, and the body regions injured simultaneously.

Fifteen players $(21 \%)$ could not accurately recall the number of injuries they sustained, let alone the details of these injuries. Of these, four overreported and 11 underreported the number of injuries sustained in the previous year. The number of injuries overreported was four, and the number of injuries underreported was 14. There was a significant association between the accuracy of retrospective recall and the number of injuries recorded prospectively $\left(\chi_{2}^{2}=14.0, p<0.001\right)$, with a higher than expected proportion of players who sustained more than one injury 


\section{Take home message}

Self reported injury history data for sports injury research cannot be relied upon with confidence. If the injury status (injured or not injured) is just being related to the outcome measure, the findings are likely to be accurate. However, requesting more detail than this-for example, number of injuries, body region injured, diagnosis-will result in a reduction in the validity of the information provided.

during the 1999 season incorrectly recalling their injury history.

\section{DISCUSSION}

The baseline risk factor questionnaire asked players to recall the number, and provide details of, any injury that was serious enough to require treatment and miss considerable amounts of playing time. Despite these wide criteria, the results of this validity study indicate that the 12 month recall of injury details at the community level of football is not entirely accurate.

Players who were not injured had no difficulty in accurately remembering this. However, previously injured players predominantly had difficulty recalling the injury diagnosis, with only $61 \%$ able to record all of their injuries in detail. A higher proportion of players were able to recall the number of injuries sustained and the body regions of the injuries.

Overall, players were able to correctly indicate their injury status (injured/uninjured) in the previous 12 months, but as the detail required and the number of injuries necessary to recall increased, the accuracy of their recall declined. Therefore, when using a self reported injury history to assess the relation between past injury and subsequent injury risk, or to establish the pattern of injuries sustained, the implications of this validity study should be considered. Reliance on self reported retrospective injury data for establishing injury patterns is likely to be inaccurate because of the inability of players to describe the number, body region injured, and diagnosis of injuries sustained. A tendency towards underreporting of injuries was also found in this validity study. Hence, self reported retrospective injury data are unlikely to provide a detailed enough description of injury patterns to inform further injury prevention research, although past injury status is reported without error over 12 months.

Comparing the past history of a specific injury with a subsequent injury must be undertaken with caution because of the less accurate recall of injury diagnosis detail and the difficulty in assessing the players' knowledge of their injury diagnoses. Comparison of provisional injury diagnoses from the prospective surveillance study with self reported injury diagnoses was made in this study. Although the provisional diagnoses were made by qualified health professionals, it is possible that these changed with subsequent investigations or clinical assessment. Therefore, whether the discrepancy seen between retrospective recollection and prospective injury records is due to a change in the diagnoses over time or incomplete recall is difficult to assess and must be acknowledged as a limitation of this study. However, the overall findings of this study show that if the injury status (injured or not injured) is just being related to the outcome measure, the findings are likely to be very accurate, but requesting more detail than this will result in a reduction in the validity of the information provided.

The findings of this validity study are consistent with those from previous populations of sporting participants ${ }^{14}$ and work related injuries. ${ }^{12}$ Community level football players were no more accurate at recalling their recent injury history than other populations despite being asked to recall only injuries meeting very specific criteria.

\section{CONCLUSION}

Overall, the findings of this small validity study suggest that community level football players more reliably recall the body region and number of injuries sustained than specific diagnoses of their injuries. Only $61 \%$ of players were completely accurate when recalling their 12 month injury history. However, a 100\% recall was shown for the past injury status - that is, yes or no. Any injury research relying on self reported injury history data to establish the relation between injury history and injury risk should consider the implications of this validity study.

\section{Authors' affiliations \\ B J Gabbe, C F Finch, University of Monash, Melbourne, Australia \\ K L Bennell, University of Melbourne, Melbourne, Australia \\ H Wajswelner, Australian Institute of Sport}

Correspondence to: Dr Gabbe, Department of Epidemiology and Preventive Medicine, Central and Eastern Clinical School, Alfred Hospital, Monash University, Commercial Road, Melbourne, Victoria, Australia 3004; belinda.gabbe@med.monash.edu.au

Accepted 26 March 2003

\section{REFERENCES}

1 Taimela S, Kujala U, Osterman K. Intrinsic risk factors and athletic injuries. Sports Med 1990;9:205-15.

2 Bennell K, Crossley K. Musculoskeletal injuries in track and field: Incidence, distribution and risk factors. Aust J Sci Med Sport 1996;28:69-75.

3 Bennell K, Wajswelner $\mathrm{H}$, Lew $\mathrm{P}$, et al. Isokinetic strength testing does not predict hamstring injury in Australian Rules footballers. Br J Sports Med 1998:32:309-14

4 Orchard J. Intrinsic and extrinsic risk factors for muscle strains in Australian football. Am J Sports Med $2001 ; 29: 300-3$

5 Voaklander D, Saunders L, Quinney H. Personal risk factors for injury in recreational and old-timer ice hockey. Sports Medicine, Training and Rehabilitation 1998:8:239-50.

6 van Mechelen W, Twisk J, Molendijk A, et al. Subject-related risk factors for sports injuries: a 1-year prospective study in young adults. Med Sci Sport Exerc 1996;28:1171-9.

7 Rothman K. Epidemiology: an introduction. New York: Oxford University Press, 2002:98.

8 Lilienfeld D, Stolley P. Foundations of epidemiology. New York: Oxford University Press, 1994:155-251.

9 Jekel J, Elmore J, Katz D. Epidemiology, biostatistics and preventive medicine. Philadelphia: WB Saunders Company, 1996:4-179.

10 McNeil D. Epidemiological research methods. Chichester: John Wiley and Sons Inc, 1996: 1-27.

11 Askling C, Lund H, Saartok T, et al. Self reported hamstring injuries in studentdancers. Scand J Med Sci Sport 2002;12:230-5.

12 Jenkins $\mathbf{P}$, Earle-Richardson G. Tucker-Slingerland D, et al. Time dependent memory decay. Am J Ind Med 2002;41:98-101.

13 Gerrard D, Waller A, Bird Y. The New Zealand Rugby Injury and Perfomance Project. II. Previous injury experience of a rugby-playing cohort. BrJ Sports Med 1994;28:229-33.

14 Twellaar M, Verstappen F, Huson A. Is prevention of sports injuries a realistic goal? A four-year prospective investigation of sports injuries among physical education students. Am J Sports Med 1996;24:528-34.

15 Kolt G, Kirkby R. Epidemiology of injury inelite and subelite female gymnasts: a comparison of retrospective and prospective findings. Br J Sports Med 1999:33:312-18.

16 Gabbe B, Finch C, Wajswelner H, et al. Australian football: injury profile at the community-level. J Sci Med Sport 2002;5:149-60.

17 Gabbe B, Finch C. A pilot case-control study to identify injury risk factors in community-level Australian football players. J Sci Med Sport 2000;3:S23-30.

18 Dawson-Saunders B, Trapp R. Basic and clinical biostatistics. London: Prentice-Hall International Inc, 1994:57-8. 
SHORT REPORT

\title{
Physiology of accidental hypothermia in the mountains: a forgotten story
}

\author{
P N Ainslie, T Reilly
}

Br J Sports Med 2003;37:548-550

Hypothermia is a serious condition, often with fatal consequences. The physiology and mechanisms of hypothermia in mountainous areas are discussed. It is as important to facilitate heat loss, especially during periods of high exertion, as it is to maintain heat production and preserve insulation. This can be partly achieved by clothing adjustments.

$\mathrm{H}$ ypothermia has long been recognised as a most serious condition, often with fatal consequences for hill walkers, fell runners, and high altitude mountain climbers. The physiology and mechanisms of hypothermia have been well documented during cold water immersion. ${ }^{1}$ However, research into hypothermia occurring in the mountainous environment has, at best, been limited. This is somewhat surprising when one considers the popularity of such mountainous pursuits. An intriguing scientific consideration therefore is entwined with the physiological considerations, and predisposing factors, of hypothermia occurring in the mountainous environment.

\section{EXERCISE INTENSITY AND FITNESS}

While walking in the mountains, participants normally operate at $30-55 \%$ of their maximal oxygen uptake, depending on the terrain. ${ }^{2}$ When exercising at these levels, the body's heat production is generally sufficient to offset heat loss. Exercise intensities (and associated energy expenditures) of this order are sufficient to prevent a fall in core temperature in wet cold conditions with mean skin temperatures as low as $20^{\circ} \mathrm{C}$ (normal mean skin temperatures are about $33^{\circ} \mathrm{C}$ ). However, groups of people walking and climbing are not always well matched physically, and, when conditions are such that mean skin temperature falls to $25^{\circ} \mathrm{C}$, there is a strong inclination to walk faster, thereby raising core temperature to a value that makes the low skin temperature tolerable. ${ }^{3}$ In this way, less fit persons may become exhausted, most likely coinciding with a decrease in body carbohydrate stores. In this sense, fitness may be important when less fit walkers pace themselves with other walkers who possess superior fitness levels. As most walkers pursue this activity in groups, the less fit walkers may be more susceptible to fatigue when exercising at a higher relative intensity compared with their fitter counterparts. The best off-set for hypothermia is to maintain heat production by means of exercise, and so fatigue becomes a critical predisposing factor.

In adverse conditions, owing to the increased insulation of clothing and hence the decreased ability to lose heat, rectal temperatures of over $39^{\circ} \mathrm{C}$ can be regularly encountered. ${ }^{2}$ The body's responses to this exercise induced "hyperthermia" are sweating and dilation of the peripheral blood vessels - that is, mechanisms to lose heat. When a rest is taken during these adverse climatic conditions, rapid cooling and a decrease in core temperature occurs. The body responds to this decrease in core temperature by constricting the peripheral vessels in an attempt to limit its heat loss. Once this vasoconstriction is maximised, core temperature can only be maintained by an increase in heat production-that is, shivering-which is thought to be the major contributor to the cold induced increase in heat production. ${ }^{5}$ However, shivering also induces vasodilation in the arterioles supplying the shivering muscles. As a result, muscle blood flow increases. The effect is that shivering reduces the insulating effect normally provided by the thickness and mass of the inactive skeletal muscle. It has been proposed ${ }^{67}$ that vasoconstricted (non-perfused) muscle acts in series with fat and skin to provide insulation, such that $75 \%$ of the body's insulation is provided by the vasoconstricted muscle and only $25 \%$ by the subcutaneous fat and skin. As a result, during exercise or shivering, the "variable" resistance of muscle vasoconstriction is lost because of increased muscle perfusion, leaving only the "fixed" resistance of the subcutaneous fat and skin. Therefore signs of shivering, in the mountainous environment, should be used as a sign of imminent danger to the participant.

\section{PROBLEM OF FATIGUE}

Once the walker, runner, or mountain climber fatigues and starts to slow or stops walking altogether, the rate of heat production falls dramatically. ${ }^{8}$ This alone predisposes to the development of hypothermia. These processes, in adverse weather conditions, will be accelerated. Likewise, if activity is pursued in conditions of hypobaric hypoxia-that is, at altitude-by virtue of a reduced inspiratory partial pressure of oxygen and hence oxygen consumption, the ability to produce heat will be decreased, further increasing the likelihood of hypothermia. The critical point for this heat loss to occur is at cessation of activity when participants take a rest or stop for fluid/food intake, during exercise when the level of exertion is low (with low heat production)-for example, when walking downhill—or during periods of navigation/group control. During these critical points, especially when participants have become wet because of adverse weather and/or high levels of exertion, heat loss will be unavoidable. ${ }^{29}$ Once the walker or runner becomes too tired to continue exercising in cold, wet, and windy conditions either because he or she is already developing hypothermia or because of the onset of exhaustion, the development of hypothermia will only be prevented if most of the following steps are undertaken. The hypothermic subject is taken rapidly to buildings or geographical features that provide shelter from the wind and rain and there is an external source of heating, a source of food, and an opportunity to change into clothes that are dry and water repellent. More clothing is required because the change from running or walking to the resting state has a considerable effect on the rate of heat production and thus the amount of clothing 
needed to maintain body temperature even at relatively mild effective temperatures. For example, clothing with at least four times as much insulation is required to maintain body temperature at rest at an effective air temperature of $0^{\circ} \mathrm{C}$ as when running at $16 \mathrm{~km} / \mathrm{h}$ at the same temperature. ${ }^{9}$ For this reason it is essential that extra clothing including water repellent outer clothing, both jacket and overtrousers, is always taken when exercising in cold and potentially wet and windy conditions. Although in the past, both poor clothing and equipment may have been factors in the increased physiological strain and likelihood of injury, ${ }^{8}$ this lack of adequate equipment cannot be considered the case today. Indeed, recent analysis of the Scottish Mountain Rescue Services indicates a decline in the number of deaths compared with previous years. ${ }^{10}$ This favourable change may reflect many developments including improvements in clothing worn by casualties, their overall skill/ awareness level, and improvements in rescue provision, particularly faster response times by the rescue teams as a result of mobile phone use. ${ }^{10}$ Despite the substantial work carried out in fabric technology, enabling greater regulation of both temperature and water vapour management in adverse weather, a continued problem is that clothing able to provide 4 CLO units of insulation may weigh as much as $10-15 \mathrm{~kg}$, making it unfeasible for general participants to carry.

\section{BEHAVIOURAL MEASURES}

During typical cold conditions, behavioural adjustments can be far more effective for maintaining thermal balance than physiological reflexes. These include all voluntary actions to increase thermal comfort. In cold climates, extra clothing is worn, exercise is undertaken, and, where possible, shelter and warmth are sought, as well as a source of food. These behavioural measures give the greatest independence from the environment and permit survival in even the most demanding of climates. However, during increased walking intensity, the clothing worn increases the evaporation required for the maintenance of thermal balance. This increase creates a difficult balance for walkers. On the one hand, they require increased insulation from clothing for protection from the environmental weather conditions. On the other hand, they need to keep the insulation relatively low to aid evaporative heat loss. The failure to achieve this balance results in increased heat strain leading to an increased likelihood of dehydration. ${ }^{11}$ From a thermoregulatory point of view, this balance will always be difficult to achieve for the walker, especially when high insulation levels are required. Furthermore, when high insulation levels are required, a situation will arise in which the clothing will restrict airflow to the skin. This restriction will promote the increased saturation of water vapour from air close to the skin. Because effective evaporation is prevented if the vapour pressure gradient between the skin and the environment is low, ${ }^{12}$ as in the case when high insulation is required, the ability of the body to evaporate water from its skin surface may become severely compromised. The significance of this decreased ability to evaporate sweat creates a paradoxical situation whereby the participant may initially, in adverse weather conditions, be at risk from increased heat strain. Therefore, in an attempt to maintain thermal balance, it is as necessary to facilitate heat loss, especially during periods of high exertion, as it is to maintain heat production and preserve insulation. By means of clothing adjustments, the anatomical areas of high heat exchange (head/face, axilla, sides of chest, groin) are areas to ventilate during activity and insulate when inactive.
Take home message

To maintain thermal balance it is as necessary to facilitate heat loss, especially during periods of high exertion, as it is to maintain heat production and preserve insulation. By means of clothing adjustments, the anatomical areas of high heat exchange (head/face, axilla, sides of chest, groin) should be ventilated as much as possible during activity and insulated during inactivity.

\section{MANAGEMENT ISSUES}

Therefore, during activity in the mountains, improper management of energy expenditure and clothing leads to sweating and fatigue. Dehydration may be a consequence of this increased rate of sweating, and, in addition to low fluid intakes, may increase this thermal stress. This likelihood of dehydration may lead to decreased thermoregulatory, circulatory, and cognitive functioning. ${ }^{11}$ In a recent study of strenuous hill walking, older age walkers were particularly prone to dehydration and decreased physical and mental performance compared with younger counterparts. ${ }^{13}$ Competitiveness may keep the walker from admitting to his or her condition, and he or she may reach a rather severe state of exhaustion and wetness before calling for a rest stop. ${ }^{414}$ At this point the individual's peripheral circulation will be dilated, so, on sitting down for a rest, heat loss occurs rapidly. ${ }^{2}$ Sweating may cease, but the wet clothing continues to dry, chilling the vasodilated vessels of the skin and adding to the increase in heat loss.

Paradoxically, it would appear that the prevention of overheating is likely to be a major factor in the prevention of hypothermia. Therefore prevention of overheating, and hence hypothermia, requires proper management of energy expenditure, clothing, fluid intake, and evaporation.

In summary, while such hypothermic events provide an intriguing scientific consideration for the environmental physiologist, such knowledge should be made applicable to both participants and the rescue services.

\section{Authors' affiliations \\ P N Ainslie, Department of Physiology and Biophysics, University of Calgary, Heritage Medical Research, Calgary, Alberta T2N 4NI, Canada \\ Thomas Reilly, Research Institute for Sport and Exercise Sciences, Liverpool John Moores University, Liverpool L3 2ET, UK}

Correspondence to: Dr Ainslie, Department of Physiology and Biophysics, University of Calgary, Heritage Medical Research Building Room 209, 3330 Hospital Drive NW, Calgary, Alberta, T2N 4NI, Canada; humpains@livjm.ac.uk

Accepted 14 January 2002

\section{REFERENCES}

1 Tipton MJ, Golden FSTC. Immersion in cold water: effects on performance and safety. In: Harries M, Williams C, Stanish WD, et al, eds. Oxford textbook of sports medicine. 2nd ed. Oxford: Oxford University Press, 1998:241-54.

2 Ainslie PN, Campbell IT, Frayn KN, et al. Physiological and metabolic responses to a hill walk. J Appl Physiol 2002;92:179-87.

3 Freeman J, Pugh LCGE. Hypothermia in mountain accidents. Int Anesthesiol Clin 1969;7:997-1007.

4 Pugh LGCE. Accidental hypothermia in walkers, climbers, and campers: report to the Medical Commission on Accident Prevention. BMJ 1966;1:123-9.

5 Doubt TJ. Physiology of exercise in the cold. Sports Med 1991;11:367-81.

6 Keatinge WR. Survival in cold water. Oxford: Blackwell Scientific, 1969.

7 Veicsteinas A, Ferretti G, Rennie DW. Superfical shell insulation in resting and exercising men in cold water. J Appl Physiol 1982;52:1557-64. 
8 Pugh LGCE. Cold stress and muscular exercise, with special reference to accidental hypothermia. BMJ 1967;2:333-7.

9 Noakes TD. Exercise and the cold. Ergonomics 2000;43:1461-79.

10 Sharp B. Strategies for improving mountain safety: analysis of Scottish mountain incidents 1996/99. Strathclyde: University of Strathclyde, 2001.

11 Sawka MN. Physiological consequences of hypohydration: exercise performance and thermoregulation. Med Sci Sports Exerc 1992;24:657-70.
12 Clark RP, Edholm OG. Man and his thermal environment. London: Edward Arnold, 1985.

13 Ainslie PN, Campbell IT, Frayn KN, et al. Energy balance, metabolism, hydration and performance during strenuous hill walking: the effect of age. J Appl Physiol 2002;93:714-23.

14 Pugh LGCE. Deaths from exposure on Four Inns Walking Competition, March 14-15, 1964. Lancet 1964;1:1281-6.

\section{Reliability of heart rate variability measures at rest and during light exercise in children}

\section{R J Winsley, N Armstrong, K Bywater, S G Fawkner}

Objectives: To investigate the reliability of heart rate variability (HRV) measures at rest and during light exercise in children.

Methods: Short term (five minute) HRV was assessed in 12 children (11-12 years of age). HRV measures were collected at rest with the children supine, breathing at 12 breaths/min, and during exercise on a cycle ergometer while exercising at $25 \%$ of peak oxygen uptake. Both resting and exercise data were collected twice from each child.

Results: Intraclass correlation coefficients were low to moderate for most measures with wide confidence intervals for each variable in both resting and exercise conditions. Random variation (typical error) within repeated measurements ranged from $31 \%$ to $187 \%$.

Conclusions: These preliminary findings suggest that HRV measures are unreliable at rest and during light exercise in children aged 11-12 years. Tighter control of extraneous influences is recommended.

$\mathrm{V}$ ariations in heart rate period arise through the balance of sympathetic and parasympathetic nervous modulation of heart rate. Through differences in the physiological response of the myocardium to the neurotransmitters acetylcholine and noradrenaline (norepinephrine), a greater variation in heart rate period arises when the parasympathetic nervous system predominates than with predominant sympathetic modulation. ${ }^{12}$ Analysis of this natural variation in heart rate period (heart rate variability (HRV)) is considered a valuable tool in providing a non-invasive window to sympathovagal regulation of heart rate. ${ }^{3}$

Time and frequency domain statistical procedures are used to analyse HRV data, ${ }^{4}{ }^{5}$ with the following measures being calculated from short term-less than five minute-heart rate recordings: in the time domain, square root of the mean of the sum of the squares of the differences between adjacent R-R intervals (RMSSD), the proportion of pairs of adjacent intervals differing by more than $50 \mathrm{~ms}$ (pNN50), and standard deviation of all the R-R intervals (SDNN); in the frequency domain, low frequency power (LF), high frequency power (HF), and total power (TP).

In adults, the HRV response to exercise has been previously documented. ${ }^{2}$ In addition, the relation of HRV to peak oxygen uptake (peak $\dot{\mathrm{V}}_{2}$ ) and physical activity levels and the effects of exercise training on HRV are widely understood, ${ }^{367}$ yet their use with children has largely focused on the neonate, ${ }^{4}$ and data on the reliability of HRV are restricted to adult subjects.

In healthy adult subjects, there exists a wide interindividual variation in HRV measures at rest. Between subject variance is reported to be in the range $12-15 \%$ for mean R-R interval, $41-155 \%$ for $\mathrm{LF}$, and $70-163 \%$ for $\mathrm{HF}$, although the cause(s) of such a wide variation is unclear. $^{8}{ }^{9}$

Within individual resting HRV measures in adults are considered reliable: RMSSD $(r=0.20-0.98)$, pNN50 $(r=0.43-0.97),{ }^{5}{ }^{10} \mathrm{HF}(r=0.48-0.96)$, LF $(r=0.60-0.97)$, and TP $(r=0.52-0.97) .{ }^{51011}$

Although group mean reliability data may indicate no significant difference between HRV measures, on an individual basis significant day to day variation exists for each person: mean (SD) variation of 23.5 (14.6)\% for pNN50 and $10.7(6.8) \%$ for SDNN. ${ }^{12}$ There appear to be no published data on the reliability of short term resting HRV measures in healthy children.

HRV measures during exercise have been investigated previously in adults. Data are sparse, but indicate that exercise HRV measurements are reliable. Tulppo et $a l^{13}$ reported the limits of agreement between repeated measures $^{14}$ during cycling at intensities of 50 and $75 \mathrm{~W}$. The range of differences for HF expressed as a coefficient of variation was $4.4 \%$. The reliability of HRV measures in children during exercise is not known and must be established to evaluate the use of this technique with young people.

The purpose of this study was therefore to assess the reliability of HRV measures in children during rest and light exercise.

\section{METHODS}

Twelve children (seven girls, five boys) volunteered for the study. Written informed consent was obtained from the participants and their legal guardians. The institutional ethics committee granted approval for the study. All children were healthy, and none were taking any prescription medications. Participants were fully habituated to equipment, protocols, and experimenters. Stature was measured using a Holtain stadiometer (Holtain, Crymych, Dyfed, UK), and body mass

Abbreviations: HRV, heart rate variability; RMSSD, square root of the mean of the sum of the squares of the differences between adjacent $R-R$ intervals; pNN50, the proportion of pairs of adjacent intervals differing by more than $50 \mathrm{~ms}$; SDNN, standard deviation of all the R-R intervals; LF, low frequency power; HF, high frequency power; TP, total power; ICC, intraclass correlation coefficient 
using Avery beam balance scales (Avery, Birmingham, UK). The children were asked to abstain from consuming caffeinated beverages and excessive physical activity in the 24 hours preceding data collection. HRV measures were collected at the same time of day.

Resting HRV data were collected on two separate days using a Polar Vantage telemetry system (Polar, Kemple, Finland), with the children in a supine position in a designated quiet room for 10 minutes. Breathing was paced at 12 breaths/min by means of a computer generated image that increased and decreased in size in rhythm with the required breathing rate, supplemented by an audible "beep" signal.

Determination of peak $\dot{\mathrm{V}}_{2}$ was performed on a different day from the collection of the resting HRV data, using an electronically braked cycle ergometer (Lode Excalibur Sport, Groningen, the Netherlands). During exercise, gas exchange variables were measured and displayed on line using a calibrated EX670 mass spectrometer (Morgan Medical Ltd, Kent, UK). Peak $\dot{\mathrm{VO}}_{2}$ was determined using a ramp test, using increments of $10 \mathrm{~W} / \mathrm{min}$, to voluntary exhaustion. Participants pedalled at a cadence of 70 (5) rpm, being actively encouraged throughout. Peak $\dot{\mathrm{V}}_{2}$ was taken as the highest recorded 10 second stationary average value during the maximal exercise test.

On separate, subsequent visits, participants completed two constant work rate exercise tests. All participants completed a six minute bout of light exercise of unloaded cycling, which represented an intensity of about $25 \%$ peak $\dot{\mathrm{V}}_{2}$. A pedal cadence of 70 (5) rpm was maintained throughout the test. Each test was completed on separate days, but at the same time of day.

HRV data were firstly cleaned using the Polar Precision Performance software set at moderate filtering level. The cleaned data were then analysed with HRV Analysis Software (Biomedical Signal Analysis Group, University of Kuopio, Finland). A continuous five minute HR recording period was selected from the resting HR data and the exercise steady state HR data. The following time domain statistics were generated: RMSSD, NN50, and pNN50. Frequency domain analysis used an autoregressive statistical method. Optimal autoregressive model order was calculated using the forwardbackward linear least squares method, ${ }^{15}$ which indicated a fixed model order of 16. To remove the influence of a large low frequency baseline trend component, detrending of the data was performed using a smoothness priors based method. ${ }^{16}$ Frequency domain statistics are presented in both $\mathrm{ms}^{2}$ and normalised units (NU). ${ }^{4}$

Paired $t$ tests and intraclass correlation coefficients (ICCs) were calculated for all HRV variables. Typical error was also calculated. ${ }^{17}$ Statistical significance was set at $p \leqslant 0.05$.

Table 1 Mean (SD) data for the two trials during resting and light exercise conditions

\begin{tabular}{llllll}
\hline & \multicolumn{2}{l}{ Rest } & & \multicolumn{2}{l}{ Light exercise } \\
\cline { 2 - 3 } \cline { 6 - 6 } \cline { 5 - 6 } & Trial 1 & Trial 2 & & Trial 1 & Trial 2 \\
\hline SDNN (ms) & $64(27)$ & $70(26)$ & & $34(20)$ & $33(20)$ \\
RMSDD (ms) & $56(28)$ & $62(27)$ & & $25(20)$ & $24(19)$ \\
NN50 (n) & $108(63)$ & $121(67)$ & & $36(60)$ & $35(57)$ \\
pNN50 (\%) & $28(19)$ & $31(18)$ & & $8(15)$ & $7(13)$ \\
LF (ms $\left.{ }^{2}\right)$ & $758(632)$ & $929(948)$ & & $440(564)$ & $422(576)$ \\
LF (NU) & $37(12)$ & $35(16)$ & $69(18)$ & $68(42)$ \\
HF (ms $\left.{ }^{2}\right)$ & $920(802)$ & $1364(1170)$ & $82(135)$ & $116(154)$ \\
HF (NU) & $53(14)$ & $55(18)$ & & $12(7)$ & $20(18)$ \\
LF/HF ratio & $0.81(0.5)$ & $0.92(1.07)$ & $8.76(6.6)$ & $8.03(8.24)$ \\
\hline
\end{tabular}

Table 2 Intraclass correlation coefficients $(r)$ with 95\% confidence intervals in parentheses for heart rate variability measures during rest and light exercise

\begin{tabular}{lll}
\hline & Rest & Light exercise \\
\hline SDNN (ms) & $0.49(-0.08$ to 0.82$)$ & $0.51(-0.09$ to 0.84$)$ \\
RMSDD (ms) & $0.44(-0.15$ to 0.80$)$ & $0.68^{*}(0.16$ to 0.90$)$ \\
NN50 (n) & $0.29(-0.31$ to 0.73$)$ & $0.79^{*}(0.39$ to 0.94$)$ \\
pNN50 (\%) & $0.20(-0.40$ to 0.67$)$ & $0.82^{*}(0.44$ to 0.95$)$ \\
LF (ms $\left.{ }^{2}\right)$ & $0.31(-0.32$ to 0.72$)$ & $0.14(-0.47$ to 0.66$)$ \\
LF (NU) & $0.37(-0.24$ to 0.76$)$ & $0.31(-0.40$ to 0.71$)$ \\
HF (ms $\left.{ }^{2}\right)$ & $0.26(-0.36$ to 0.70$)$ & $0.76^{*}(0.32$ to 0.93$)$ \\
HF (NU) & $0.44(-0.17$ to 0.79$)$ & $0.65^{*}(-0.19$ to 0.81$)$ \\
LF/HF ratio & $0.14(-0.47$ to 0.62$)$ & $0.28(-0.36$ to 0.73$)$ \\
\hline${ }^{*} p \leqslant 0.05$. & &
\end{tabular}

\section{RESULTS}

Data were collected from 12 children, mean (SD) age 12.8 (0.3) years, mean (SD) body mass 46.6 (10.1) kg, and mean (SD) stature 1.56 (0.06) m. Group mean peak oxygen uptake was 41 (6) $\mathrm{ml} / \mathrm{kg} / \mathrm{min}$. Constant load exercise was performed at an intensity of $25(4) \%$ peak $\dot{\mathrm{V}}_{2}$.

At rest and during light exercise there were no significant differences between the group mean values between trial 1 and 2 for any time domain or frequency domain HRV variable (table 1). None of the ICCs for resting measures were significant $(\mathrm{p} \geqslant 0.05$; table 2$)$, however, during light exercise ICCs for RMSSD, NN50, pNN50, and HF were all significant $(p \leqslant 0.05)$. Calculation of typical error indicated a random variation in the results of $31-187 \%$ (table 3 ).

\section{DISCUSSION}

HRV analysis is a novel research tool in exercise and sports medicine, ${ }^{4}$ yet before this work can be extended to children it is imperative to establish the reliability of such measures within that population. Determination of reliability is a composite measure that provides information on the stability, accuracy, and size of measurement error of a dependent variable. By minimising measurement error and controlling for influencing factors, the stability and accuracy of the dependent variable can be better determined. The benefits of ensuring reliability are that the resultant data can be interpreted in a meaningful way, the measurement tool/ procedure can be replicated with confidence by others, and the experimental conditions used have suitably attenuated the effects of any confounding variables. ${ }^{18}$

Although there were no significant differences between the group means between trial 1 and 2 for any HRV variable, the standard deviations of these mean scores were large. The ICCs were lower than those reported for adults. ${ }^{5} 10^{11}$ In addition, most of the ICCs were non-significant or had associated wide $95 \%$ confidence intervals (table 2). Hopkins ${ }^{17}$

Table 3 Typical error expressed as a coefficient of variation (\%) of the differences between the two trials for resting and light exercise conditions

\begin{tabular}{lll}
\hline & Rest & Light exercise \\
\hline SDNN (ms) & 31 & 45 \\
RMSDD (ms) & 37 & 59 \\
NN50 (n) & 72 & 179 \\
pNN50 (\%) & 81 & 187 \\
LF $\left(\mathrm{ms}^{2}\right)$ & 97 & 142 \\
LF (NU) & 38 & 33 \\
HF (ms ${ }^{2}$ ) & 84 & 143 \\
HF (NU) & 35 & 75 \\
LF/HF ratio & 83 & 74 \\
\hline
\end{tabular}




\section{Take home message}

These preliminary data suggest that the reliability of heart rate variability measures at rest and during light exercise in children is weak. Further research is required to determine whether reliability can be improved with tighter control of extraneous variables.

argues that calculating typical error gives a more informative reliability statistic than simply calculating differences and ICCs. Typical error represents how much of the change in scores is due to random variation-this random variation may arise through both day to day variation (biological variation) and through measurement error. The typical error statistic for every HRV variable indicated the presence of a sizeable random variation (31-187\%) (table 3), and it is this randomness of the resultant HRV data that may help to explain the large standard deviations, low ICCs, and wide 95\% confidence intervals observed with these data.

Although attempting to control for the potentially confounding effects of drugs, previous activity, and caffeinated beverages on HRV in these children, differences in preparations before measurement may have occurred, leading to the significant day to day variations seen. For example, it is well documented that children are poor at recalling previous physical activity, and their perception of exercise intensity is more inconsistent than that of adults. ${ }^{19}$ Consequently, it is difficult to guarantee that all the children avoided excessive exercise on the days before data collection. In addition, although breathing rate was held constant during resting HRV measures, tidal volume, which also influences HRV in children, ${ }^{20}$ was not. These factors, combined with the small sample size, may have colluded to produce the wide variation seen.

These data suggest that the reliability of HRV measures in children is unconvincing, therefore researchers and clinicians should be cautious in using and interpreting them. Whether such reliability could be improved with tighter control of potentially confounding variables warrants further investigation.

\section{ACKNOWLEDGEMENTS}

The results were analysed with HRV Analysis Software 1.1 for windows developed by The Biomedical Signal Analysis Group, Department of Applied Physics, University of Kuopio, Finland.

\section{Authors' affiliations}

R J Winsley, Children's Health and Exercise Research Centre, University of Exeter, Heavitree Road, Exeter EX1 2LU, Devon, UK

N Armstrong, K Bywater, S G Fawkner, University of Exeter

Correspondence to: Dr Winsley, University of Exeter, Children's Health and Exercise Research Centre, Heavitree Road, Exeter EXI 2LU, Devon, UK; r.j.winsley@exeter.ac.uk

Accepted 16 June 2003

\section{REFERENCES}

1 Mokrane A, Nadeau R. Dynamics of heart rate response to sympathetic nerve stimulation. Am J Physiol 1998;275:H995-1001.

2 Rowell LB. Human cardiovascular control. Oxford: Oxford University Press, 1993.

3 Hainsworth R. The control and physiological importance of heart rate. In: Malik M, Camm AJ, eds. Heart rate variability. New York: Futura Publishing Company, 1995:3-19.

4 Camm AJ, Malik M, Bigger JT, et al. Task force of the European Society of Cardiology and the North American Society of Pacing and Electrophysiology. Heart rate variability: standards of measurement, physiological interpretation and clinical use. Circulation 1996;93:1043-65.

5 Pitzalis MV, Mastropasqua F, Massari F, et al. Short-term and long-term reproducibility of time and frequency domain heart rate variability measurements in normal subjects. Cardiovasc Res 1996;32:226-33.

6 Jensen-Urstad K, Saltin B, Ericson M, et al. Pronounced resting bradycardia in male elite runners is associated with high heart rate variability. Scand J Med Sci Sports 1997; 7:274-8.

7 Tsuji H, Larson MG, Venditti FJ, et al. Impact of reduced heart rate variability on risk for cardiac events. Circulation 1996;94:2850-5.

8 Abdel-Rahman ARA, Merrill RH, Wooles WR. Gender related differences in baroreflex control of heart rate in normotensive humans. J Appl Physiol 1994;77:606-13.

9 Huikuri HV, Pikkujamsa SM, Airaksinen KEJ, et al. Sex related differences in autonomic modulation of heart rate in middle aged subjects. Circulation 1990;94:122-5.

10 Melanson EL. Resting heart rate variability in men varying in habitual physical activity. Med Sci Sports Exerc 2000;32:1894-901.

11 Hohnloser SH, Klingenheben T, Zabel M. Intraindividual reproducibility of heart rate variability. Pace 1992;15:2211-14.

12 Kautzner J. Reproducibility of heart rate variability measurement. In: Malik M, Camm AJ, eds. Heart rate variability. New York: Futura Publishing Company, 1995: 165-71

13 Tulppo MP, Mäkikallio TH, Seppänen T, et al. Vagal modulation of heart rate during exercise: effects of age and physical fitness. Am J Physiol 1998;274:H424-9.

14 Bland JM, Altman DG. Statistical methods for assessing agreement between two methods of clinical measurement. Lancet 1986;1:307-10.

15 Niskanen JP, Tarvainen MP, Ranta-Aho PO, et al. Software for advanced HRV analysis. Kuopio: University of Kuopio Press, 2002.

16 Tarvainen MP, Ranta-Aho PO, Karjalainen PA. An advanced detrending method with application to HRV analysis. IEEE Trans Biomed Eng 2002;49:172-5

17 Hopkins WG. Measures of reliability in sports medicine and science. Sports Med 2000;30:1-15.

18 Vincent WJ. Statistics in kinesiology. Champaign, IL: Human Kinetics, 1999.

19 Armstrong N, Welsman JR. Young people \& physical activity. Oxford: Oxford University Press, 1997.

20 Williams $\mathrm{C}$, Lopes $\mathrm{P}$. The influence of ventilatory control on heart rate variability in children. J Sports Sci 20:407-15. 